\title{
Letter
}

\section{Structural Differences in the Analysis of Mechanical and Electrical Systems}

\author{
Parfentiev Nikolay Andreevich $^{1}$, Parfentieva Natalia Andreevna $^{2}$, Truhanov StepanVinokenievich ${ }^{2}$ \\ ${ }^{1}$ All-Russian Research Institute for Optophysical Measurements (VNIIOFI), Moscow, Russia \\ ${ }^{2}$ Departamebt of Physics, Moscw State University of Civil Engineering, Moscow, Russia
}

Email address:

Nik_parfenium@mail.ru (P. N. Andreevich)

\section{To cite this article:}

Parfentiev Nikolay Andreevich, Parfentieva Natalia Andreevna, Truhanov StepanVinokenievich. Structural Differences in the Analysis of Mechanical and Electrical Systems. American Journal of Embedded Systems and Applications. Vol. 6, No. 1, 2018, pp. 11-14.

doi: 10.11648/j.ajesa.20180601.12

Received: April 25, 2018; Accepted: May 22, 2018; Published: June 11, 2018

\begin{abstract}
Mechanical systems, the description of which differs from the electrical circuits of similar structure, are studied. As an example, the scheme of a spring pendulum with a zero periodic displacement of the point of application of the forcing harmonic force is considered. In contrast to the unique representation of electrical circuits mechanical system with a serial connection of mechanical elements can be modeled by different electrical circuits. In this case, the family of frequency characteristics of such a system contains a singular point, which is at a frequency $\sqrt{2}$ less than the resonance frequency. The force modulus at this point does not depend on the value of the viscous resistance.
\end{abstract}

Keywords: Simulation of Systems, The Method of Analogies, Mechanical Resonance

\section{Introduction}

In the sections of mechanics and electrical engineering the General scheme for the analysis of a spring pendulum and an oscillating circuit is usually applied. In some textbooks the parallel and serial connection of springs is considered by analogy with connection of capacitors and resistors. At present, the calculation of mechanical systems is carried out mainly with the help of computer programs [1-3]. At the same time, the physical basis for the correspondence of the mechanical system and its model is often left out. In particular, it can be used to describe all variants of the scheme of the spring pendulum, and the section of forced oscillations of the mechanical resonator can be described in the manner of describing the alternating current in electrical engineering. A possible reason for limiting the use of the method of electromechanical analogies is the desire to first determine the mechanical displacement (the most obvious) while, with a single approach, the speed is a direct analogue of the alternating current in electrical engineering. In this article, an attempt is made to present the mechanics section in a uniform manner, producing in some cases paradoxical and unusual results.

\section{An Example of a Mechanical System That Does Not Have a Direct Electric Analog}

There are fundamental differences in the modeling of mechanical and electrical systems.

As an example, consider the scheme of the spring pendulum (Figure 1), in which there are no friction forces - the load is on a perfectly smooth surface.

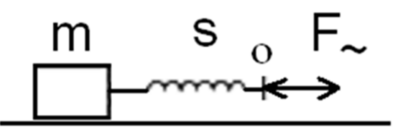

Figure 1. Scheme of the ideal spring pendulum.

In the case of an ideal spring (zero mass and no wave processes), the forces applied to the spring and the load are equal to the external force $\mathrm{F} \sim$.

The displacement of the point $\mathrm{O}$ will be equal to the sum of the spring compression and movement of cargo, which 
naturally means the summation of the velocity of the spring compression $-V_{s}$ and the velocity of the cargo- $V_{m}$. The velocity of the $\mathrm{O}$-point can be determined from the equation

$$
V_{o}=\int \frac{F}{m} d t+\frac{d F}{k d t}
$$

In the case of periodic force $F_{\sim}=F_{o} \sin (\omega t)$, it is easy to find the ratio between the amplitudes of force and velocity

$$
V_{o}=F_{o}\left(\frac{\omega}{k}-\frac{1}{\omega m}\right)
$$

Paradoxically, the velocity and periodic displacement of the O-point are equal to zero at the resonant frequency. In this case, the cargo will fluctuate under the influence of periodic force. In the general case, the action of a periodic force on the system under consideration will cause motion with a constant velocity, but the point $\mathrm{O}$ will not perform periodic oscillations. At the initial phase of the periodic force, equal to $\frac{\pi}{2}$ or $\frac{3 \pi}{2}$ constant velocity of the system will be zero. The amplitude of the periodic velocity of the load is equal $V_{m}=\frac{F}{m \omega}$, and the amplitude of displacement $-x_{m}=\frac{F}{m \omega^{2}}$.

The formula for the speed of the load can be rewritten as

$$
V_{o}=\frac{F_{o}}{\frac{1}{\frac{\omega}{k}-\frac{1}{\omega m}}}
$$

Thus, to determine the speed in this system, the amplitude of the force must be divided by the amount of the inverse sum of the mechanical resistance of the mass and the reverse mechanical resistance of the spring.

Algorithm for calculating the mechanical resistance of the mechanical system, which is a parallel connected mass (reactive resistance -) and a spring (reactive resistance $-\frac{k}{\omega}$ ), is implemented. For full compliance with the electrical circuits in the method of electromechanical analogies is used the flexibility of the spring is equal to the reciprocal of stiffness.

If the cargo under motion experiences viscous friction (i.e. there is a friction force proportional to the speed of its movement) mechanical resistance of the cargo is equal to the sum of the reactive resistance of the cargo and the active coefficient of viscous friction $-R=\frac{F}{V}$ ).

As a result, the mechanical system with a serial connection of the spring and the load depending on the method of applying the force is modeled by various electrical circuits.

The considered version of the scheme has no direct analogues in electrical engineering. The strength and speed of the mechanical system correspond to the voltage and current. It is impossible to imagine connecting a series-connected capacitor and inductor to a voltage source so that the currents in these elements are summed.

At the same time in mechanics there are systems with parallel connection of elements which are modeled by various electric circuits (Figure 2).
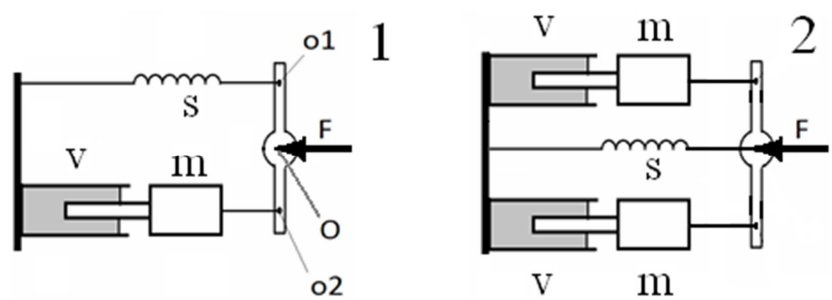

Figure 2. Mechanical systems with parallel connection of elements. The system 1 is simulated by a parallel electric circuit, 2 - serial.

The essential difference between the system 1 (Figure 2) it is that the weightless lever contains three hinges $(0, \mathrm{O} 1$ and $\mathrm{O} 2$ ). As a result, the subsystems ( $\mathrm{m}$ and $\mathrm{s}$ ) have the same equal force, and the displacement of the point of application of force is equal to the sum of the displacement of the subsystems.

When describing the section of forced oscillations of mechanical systems, excitation of oscillations under the action of impulse force is usually stated, since it is very difficult to practically implement a system with a force varying according to the harmonic law, applied to the mobile part of the mechanical system.

However, it is relatively easy to implement a system in which the constant displacement of a particular part changes the speed of its movement. This is the so - called crank mechanism with a relatively longer (compared to the diameter of the wheel) lever. Changing the speed of the wheel leads to pseudoharmonic movements of the connecting rod. This mechanism is used in the demonstration system, a full analysis of which is given below.

For the future, it is important that there is a mechanism that can be used as a source of velocity linearly increasing with frequency $V=x_{0} \omega$.

\section{Analysis of the System with a Source of Linearly Increasing Velocity}

When connecting such a source to the considered system with viscous friction, the problem of determining the speed of the load is reduced to finding the current in one of the two parallel branches connected to the speed source discussed above. The scheme of this calculation is shown in Figure 3.

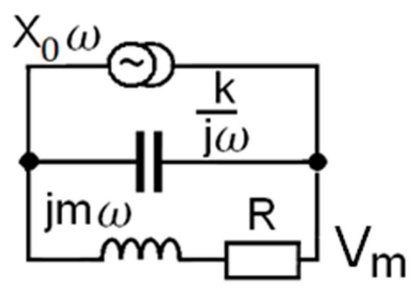

Figure 3. Equivalent circuit of the mechanism (Figure 1) with the source velocity and the viscous friction of the load.

For figure 4 the dependence of the relative velocity of the load on the relative frequency and the normalized viscous resistance is given. 


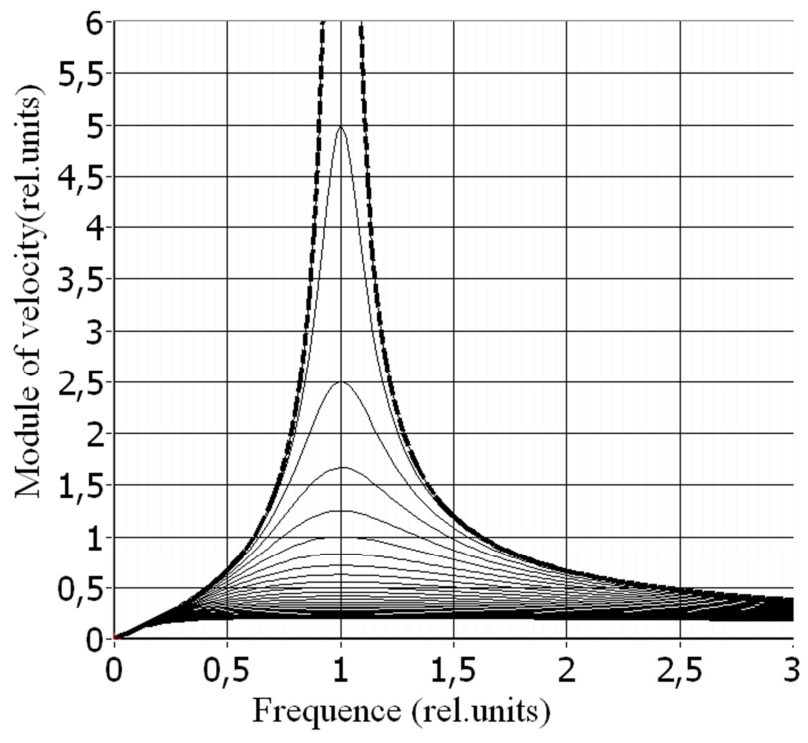

Figure 4. Dependence of the relative velocity of the load on the relative frequency and the normalized value of the viscous resistance.

It can be noted that the maximum forced velocity of the load oscillations occurs at a resonant frequency at any values of the relative viscosity coefficient. The asymptotes of the family of frequency characteristics are the abscissa axis and the function $\left|\frac{\bar{\omega}}{1-\bar{\omega}^{2}}\right|$, where $\bar{\omega}$ the ratio of the frequency to the resonance frequency in the absence of friction.

For figure 5 the dependence of the relative amplitude of the forced displacement of the load depending on the relative frequency and the normalized viscous resistance is presented.

It can be noted that the maximum amplitude of the forced oscillations is shifted to the low frequency region with an increase in the coefficient of viscous friction. The asymptotes of the family of frequency characteristics of the function $\left|\frac{1}{1-\bar{\omega}^{2}}\right|$ and the broken line composed of a horizontal axis and cut the $\mathrm{y}$-axis from 0 to 1 .

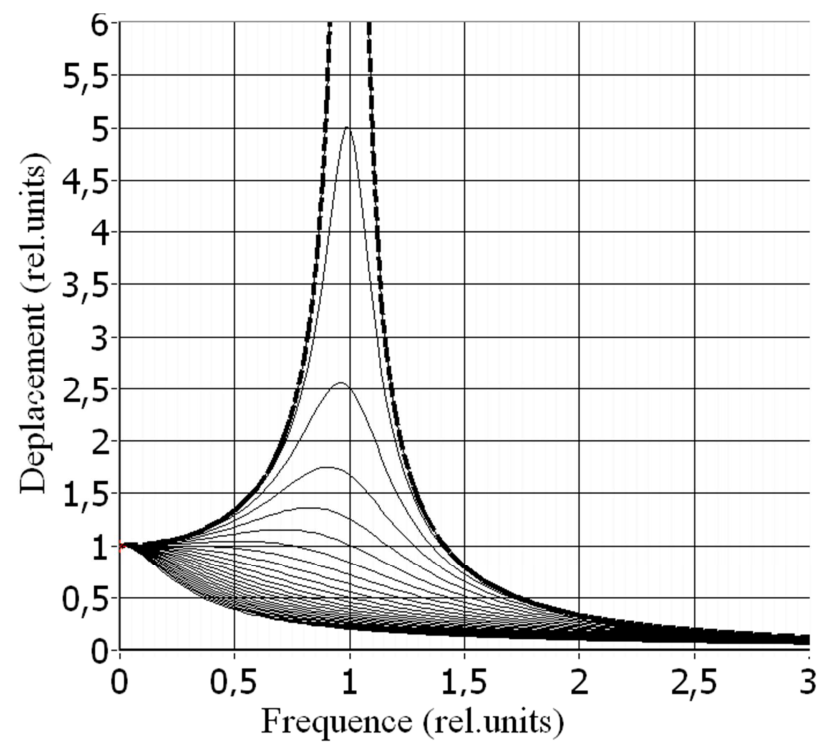

Figure 5. Dependence of the relative amplitude of the forced displacement of the load depending on the relative frequency and the normalized viscous resistance.
It is also possible to calculate the amplitude of the force applied to the considered mechanical system, multiplying the speed of the oscillation source by the total mechanical resistance. The results of calculations are shown in Figure 6.

Note, that the maximum amplitude of the force of the driving vibrations is shifted to the high frequency region by increasing the coefficient of viscous friction. Asymptotes of the family of frequency characteristics are function $\left|\frac{\bar{\omega}^{2}}{1-\bar{\omega}^{2}}\right|$ and broken composed of a line $Y=1$ and a segment of the ordinate axis from 1 to 0 .

A remarkable feature of this family of curves is the existence of a singular point at a relative frequency, where the modulus of force does not depend on the magnitude of the viscous resistance. The existence of this point in the characteristics of different types of electric circuit was previously reported $[4,10]$

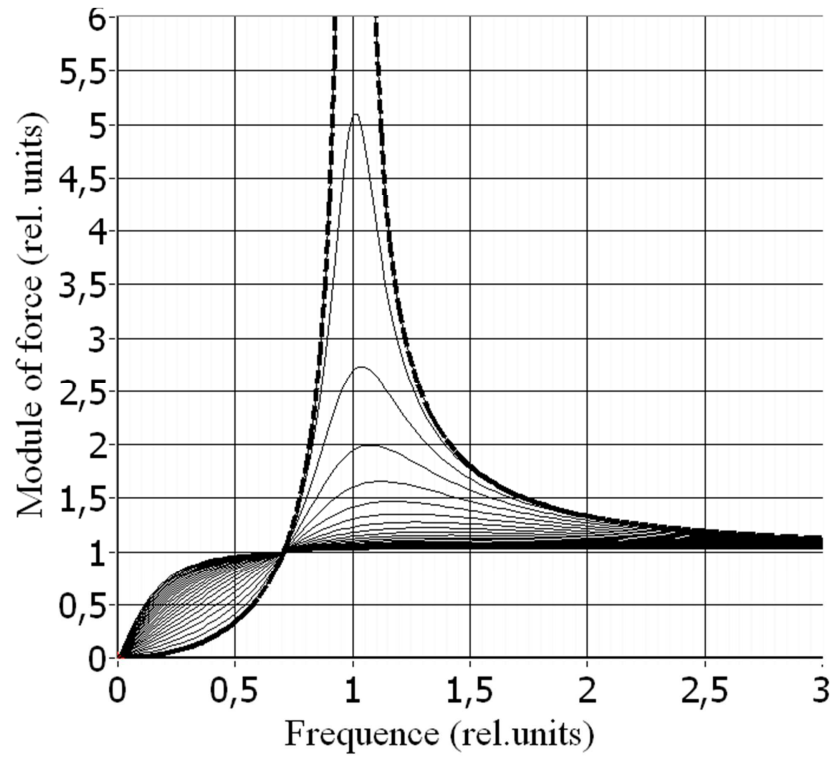

Figure 6. Dependence of the normalized power module on the frequency and normalized viscous resistance.

For example, forced oscillations of mechanical systems in physics textbooks are often used significantly more complex mechanical system, the scheme of which is shown in Figure 6. It is easy to analyze, but it is hardly suitable for users just beginning their acquaintance with mechanical systems and their forced oscillations.

For (figure7) the equivalent circuit of a mechanical system is also presented.
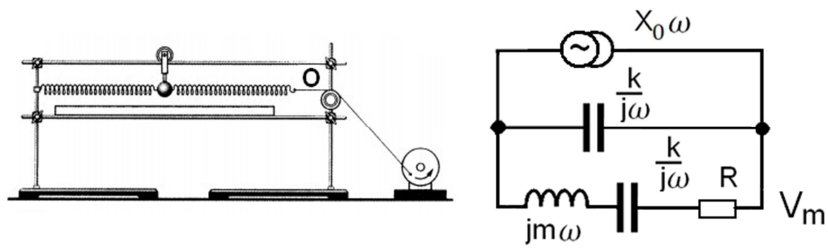

Figure 7. Installation Diagram for demonstration of forced oscillations and design diagram of the installation.

The Point $\mathrm{O}$ moves with a constant amplitude linearly 
increasing from the frequency speed $-x_{0} \omega$. The velocity of the ball in the absence of viscous friction can be defined as

$$
V_{\mathrm{M}}=\frac{x_{0} \omega \frac{k}{i \omega}}{\frac{2 k}{i \omega}+i \omega m}
$$

Where $\mathrm{k}$ is the stiffness coefficient of a single spring, and the $x_{0}$ - strain amplitude. After reduction to the normalized frequency we receive

$$
V_{\mathrm{M}}=\frac{x_{0} \omega_{0} \bar{\omega}}{2-\bar{\omega}^{2}}
$$

Therefore, the resonance frequency corresponds to the parallel connection of two springs between which the ball is located.

Taking into account the viscous friction allows to determine the dependence of the amplitude of the velocity and displacement of the ball on the frequency and relative viscosity.

These dependences coincide with the characteristics shown in Figure 4 and 5 with the only difference that the resonant frequency of the system is at times $\sqrt{2}$ higher than the resonant frequency of the ball and a separate spring. This is obvious, because with this frequency oscillates the ball placed between two identical springs.

For Figure 8 the frequency dependence of the normalized $\left(k x_{0}\right)$ force acting on the analyzed mechanical system is given.

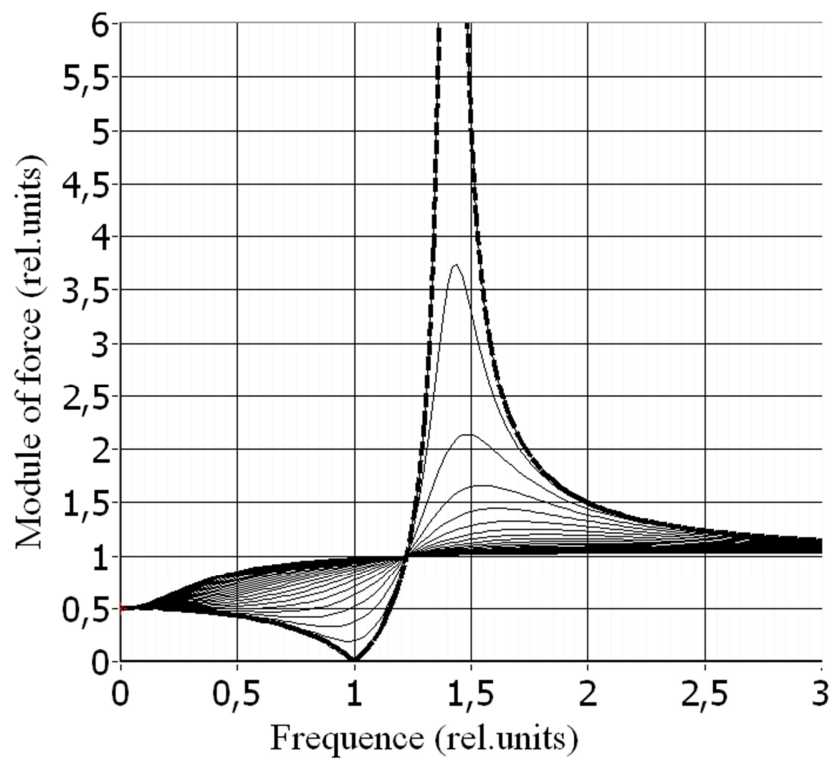

Figure 8. Dependence of the rated power on the frequency for the installation shown in Figure 6.

Asymptotes of the family of frequency characteristics are function $\mathrm{a}\left|\frac{1-\bar{\omega}^{2}}{2-\bar{\omega}^{2}}\right|$ nd broken composed of a line $Y=1$ and a segment of the ordinate axis from 0.5 to 1 .

In the family of characteristics (t.8) there is a point with a zero value of force corresponding to the successive resonance of the system with the load and the spring connected to it.

The unit for demonstration of forced oscillations can be constructed according to a simpler and more obvious scheme. For this purpose it is necessary to remove the second spring (attached to a fixed point), and to connect the first spring to the crank mechanism driven by the disk of the motor already available in the old installation.

\section{Conclusion}

It is noted the main difference modeling of mechanical systems consists in the fact that the scheme of connection of its elements (electric completely determines the model) requires further analysis. Mechanical systems are much more diverse than electrical systems. It is shown that some of them do not have a direct electric analog. We consider in detail one of such systems, the frequency response of which contains a singular point.

\section{References}

[1] Ivanov V. (et al) Advanced in Design Simulation and Manufacturing. Springer 2018.

[2] Friedrich Pfeiffer The art of modeling Mechanical Systems Springer 2017.

[3] Alexander N. Evgrafov Advanced in Mechanical Engineering Saint Petersburg, Russia, 2016.

[4] Parfentyev N. A., Parfentyeva E. N., Sevastyanov S. I. Pecularity of frequency characteristics of a parallel oscillatory contour. News of higher education institutions. Electronics No. 62014, page 110 .

[5] Parfentyev N. A. Invariant one model of a mechanical system with viscous friction, Electronic journal technical acoustics. 2014.

[6] Parfentyev N. A., Parfentyeva N. A., Truchanov S. V.. On the singular point of the frequency characteristics of oscillatory systems. Natural and technical Sciences, №9-10, 2014, c. 31.

[7] Parfentyev N. A., Parfentyeva E. N., Krivolutsrfya N. V. Amazing properties of a parallel electrical circuit. XIX International Scientific and Practical Conference. Russia St. Petersburg, 19-20.02.2016 page.11-15.

[8] Parfentev N. A. New paradoxes the model of an RLC circuit. Science of Europe. N12. Vol. 1, page 19-23.

[9] Parfentev N. A. Paradoxes the Model of an RLC Circuit. Engineering Physics Volume 2, Issue 3, June 2017, Pages: 49-53.

[10] Parfentev N. A., Parfentyeva N. A., A Singular Point in the Characteristics of the Serial RLC Circuit. Journal of Physics and Applications._Vol. 5, No. 6, 2017, pp. 109-112. 